\title{
Flexural Behavior of Laterally Loaded Tapered Piles in Cohesive Soils
}

\author{
Musab Aied Qissab \\ Department of Civil Engineering, Al-Nahrain University, Baghdad, Iraq \\ Email: musstruct@nahrain-eng.org \\ Received 16 January 2015; accepted 6 February 2015; published 10 February 2015 \\ Copyright (C) 2015 by author and Scientific Research Publishing Inc. \\ This work is licensed under the Creative Commons Attribution International License (CC BY). \\ http://creativecommons.org/licenses/by/4.0/

(c) (i) Open Access

\begin{abstract}
In this paper, the flexural behavior of laterally loaded tapered piles in cohesive soils is investigated. The exact solution for the governing differential equation of the problem is obtained based on the beam-on-elastic foundation approach in which the soil reaction on the pile is related directly to the pile lateral deflection. In this investigation, the modulus of subgrade reactions is assumed to be constant along the pile depth. Parametric study through numerical examples is carried out to prove the validity and accuracy of the obtained results. In general, the derived displacement field can be used to study pile response in multilayered soil profiles by subdividing the pile into a number of elements. It is found that tapered piles show stiffer behavior than that for prismatic ones having the same material volume with an optimum stress distribution along the pile depth. Accordingly, tapered piles are more efficient and economic than those having the same material volume. Verification is also carried out for the obtained results through finite element analysis and the selected number of elements gives a very good agreement for lateral deflection and a larger number of elements is required to obtain better results for bending moment because of moment loss resulting from the lack of shear diagram.
\end{abstract}

\section{Keywords}

Cohesive Soils, Flexural Behavior, Lateral Load, Subgrade Reaction, Tapered Piles

\section{Introduction}

Piles are widely used to support structures not limited to bridges, high rise buildings and offshore structures which are subjected toaxial and lateral loads resulting from different sources. Tapered piles, as special cases, have received great attention at present due to their good performance in resisting loads in comparison to that for prismatic ones because of the optimum material distribution with respect to loading intensity. Most of the avail- 
able analysis and design guidelines lay more emphasis on prismatic piles over tapered piles despite of the economical advantage of the latter. Tapered piles are not widely used as a design option because of the limit knowledge about their behavior under different loading types in comparison to the prismatic piles.

There are a number of studies concerning the behavior of individual piles. Wei [1] studied experimentally the static behavior of piles in cohesionless soils under the effect of axial, lateral, and cyclic loads. Two sets of tests with three types of geometries including a prismatic pile in dry sandy soil were conducted to study their behavior. The results of the study confirmed the efficiency of tapered piles over the prismatic ones having the same material input.

Horvath et al. [2] investigated experimentally the behavior of tapered tube piles under axial, uplift, and lateral loads in sand. The experimental program was mainly conducted for one of the larger transportation projects for the major renovation and expansion of John F. Kennedy International Airport in New York City to verify the performance of these piles. It was established from the experimental results that taper-tube piles are successfully resist the entire spectrum of axial and lateral loads that is normally encountered in transportation engineering.

Shankar et al. [3] developed a procedure to predict the flexural behavior of axially loaded and laterally loaded tapered piles embedded in liquefaction-induced laterally spread soils. The problem was analyzed by using the modulus of subgrade reaction approach based on Winkler type soil model. The resulting governing equation to solve the flexural behavior of the pile with the specified boundary conditions was solved by using finite difference technique. The use of tapered piles was found beneficial in liquefaction-induced laterally spreading soils as the maximum bending moment developed due to drag force is less especially when the applied axial force is much lower than the critical load.

Zhan et al. [4] studied the load capacity behavior of two series of axially loaded tapered piles in sand by using finite element method. It was observed from the numerical analysis that the shaft resistance increasing with the tapered angle with an increase of (12\%) over that of the straight-side piles at an optimum tapered angle. It was concluded that tapered piles are more suitable for floating pile foundations.

\section{Statement of the Problem}

The tapered pyramidal pile shown in Figure 1 of length $(L)$ and embedded in a homogeneous cohesive soil layer. is subjected at its head to a lateral concentrated load $(Q)$ and a bending moment $(M)$.

The governing differential equation of the above problem was given by Hetenyi [5] for beams on elastic foundation with variable flexural rigidity as follows:

$$
\frac{\mathrm{d}^{2}}{\mathrm{~d} z^{2}}\left[E I_{(z)} \frac{\mathrm{d}^{2} y}{\mathrm{~d} z^{2}}\right]+k_{z} y=0
$$

Or

$$
E\left[I_{(z)} \frac{\mathrm{d}^{4} y}{\mathrm{~d} z^{4}}+2\left(\frac{\mathrm{d} I_{(z)}}{\mathrm{d} z}\right) \frac{\mathrm{d}^{3} y}{\mathrm{~d} z^{3}}+\left(\frac{\mathrm{d}^{2} I_{(z)}}{\mathrm{d} z^{2}}\right) \frac{\mathrm{d}^{2} y}{\mathrm{~d} z^{2}}\right]+k_{z} y=0
$$

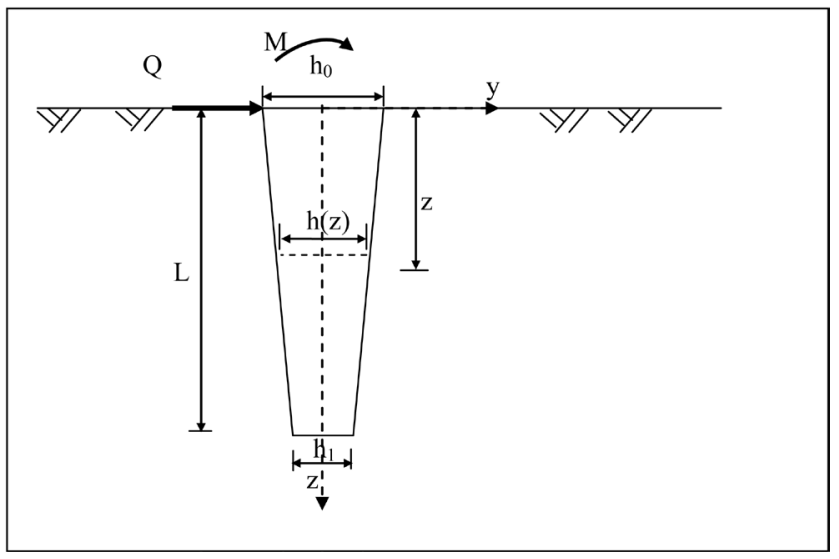

Figure 1. The considered pile configuration. 
where $k_{z}$ is the modulus of subgrade reaction $=K b(z)$, and $E$ is the modulus of elasticity of the pile material. The moment of inertia $I(z)$ can be given by:

$$
I_{(z)}=\frac{b_{(z)}\left(h_{(z)}\right)^{3}}{12}=\frac{b_{0} h_{0}^{3}}{12}(1-c z)^{4}=I_{0}(1-c z)^{4}
$$

in which:

$b_{(z)}=b_{0}(1-c z) ; h_{(z)}=h_{0}(1-c z) ; b_{0}, h_{0}$ are the section dimensions of the pile at $z=0 ; c=\left(h_{0}-h_{1}\right) /\left(h_{0} L\right) ;$ $h_{1}=$ the pile dimension in the direction of $y$ at $z=L$; and $I_{0}$ is the moment of inertia at $z=0$.

Substituting Equation (3) into (2) and assuming that $\bar{Z}=(1-c z)$ and after simplifications, the following equation can be obtained:

$$
c^{4} \bar{z}^{4} \frac{\mathrm{d}^{4} y}{\mathrm{~d} \bar{z}^{4}}+8 c^{4} \bar{z}^{3} \frac{\mathrm{d}^{3} y}{\mathrm{~d} \bar{z}^{3}}+12 c^{4} \bar{z}^{2} \frac{\mathrm{d}^{2} y}{\mathrm{~d} \bar{z}^{2}}+\frac{k_{z}}{E I_{0}} y=0
$$

The solution of Equation (4) can be expressed as follows:

$$
y(z)=A_{1} \bar{Z}^{\left(a_{1}+b_{1}(i)\right)}+A_{2} \bar{Z}^{\left(a_{2}+b_{2}(i)\right)}+A_{3} \bar{Z}^{\left(a_{3}+b_{3}(i)\right)}+A_{4} \bar{z}^{\left(a_{4}+b_{4}(i)\right)}
$$

or

$$
y(z)=A_{1} \bar{z}^{\left(a_{1}\right)} \mathrm{e}^{i\left(b_{1} \ln (\bar{z})\right)}+A_{2} \bar{z}^{\left(a_{2}\right)} \mathrm{e}^{i\left(b_{2} \ln (\bar{z})\right)}+A_{3} \bar{z}^{\left(a_{3}\right)} \mathrm{e}^{i\left(b_{3} \ln (\bar{z})\right)}+A_{4} \bar{z}^{\left(a_{4}\right)} \mathrm{e}^{i\left(b_{4} \ln (\bar{z})\right)}
$$

where

$$
\begin{aligned}
& a_{1}=\frac{1}{2}(-1-a), b_{1}=-\frac{\bar{\beta}}{a} \\
& a_{2}=\frac{1}{2}(-1+a), b_{2}=\frac{\bar{\beta}}{a} \\
& a_{3}=\frac{1}{2}(-1-a), b_{3}=\frac{\bar{\beta}}{a} \\
& \left.a_{4}=\frac{1}{2}(-1+a), b_{4}=-\frac{\bar{\beta}}{a}\right) \\
& a=\sqrt{\frac{5}{2}\left[1+\sqrt{1+\left(\frac{4}{5} \bar{\beta}\right)^{2}}\right]}, \bar{\beta}=\sqrt{\frac{\beta^{4}}{c^{4}}-1}, \quad \beta=\frac{k_{z}}{E I_{0}} \text {, and } \bar{Z}=(1-c z)
\end{aligned}
$$

The constants $A_{1}, A_{2}, A_{3}$, and $A_{4}$ can be determined by applying the boundary conditions as follows: at $(z=0)$

$$
\begin{gathered}
\frac{\mathrm{d}^{2} y}{\mathrm{~d} z^{2}}=\frac{M}{E I_{0}} \\
E\left[I_{(z)} \frac{\mathrm{d}^{3} y}{\mathrm{~d} z^{3}}+\frac{\mathrm{d} I_{(z)}}{\mathrm{d} z} \frac{\mathrm{d}^{2} y}{\mathrm{~d} z^{2}}\right]=Q
\end{gathered}
$$

or

$$
\left[(\bar{z}) \frac{\mathrm{d}^{3} y}{\mathrm{~d} z^{3}}-4 c \frac{\mathrm{d}^{2} y}{\mathrm{~d} z^{2}}\right]=\frac{Q}{E I_{0}}
$$

at $(z=L)$

$$
\frac{\mathrm{d}^{2} y}{\mathrm{~d} z^{2}}=0
$$




$$
\left[(\bar{z}) \frac{\mathrm{d}^{3} y}{\mathrm{dz}}-4 c \frac{\mathrm{d}^{2} y}{\mathrm{dz^{2 }}}\right]=0
$$

Applying the above boundary conditions, the following equations can be obtained: at $(z=0)$

$$
\begin{aligned}
& \left(\bar{z}^{a_{1}} D_{21}-2 a_{1} c D_{11}+a_{1}\left(a_{1}-1\right) c^{2} D_{01}\right) A_{1}+\left(\bar{z}^{a_{1}} D_{22}-2 a_{1} c D_{12}+a_{1}\left(a_{1}-1\right) c^{2} D_{02}\right) A_{2} \\
& +\left(\bar{z}^{a_{2}} D_{21}-2 a_{2} c D_{11}+a_{2}\left(a_{2}-1\right) c^{2}\right) A_{3}+\left(\bar{z}^{a_{2}} D_{21}-2 a_{2} c D_{11}+a_{2}\left(a_{2}-1\right) c^{2}\right) A_{4}=M /\left(E I_{0}\right) \\
& \left(\bar{z} D_{311}-4 c D_{211}\right) A_{1}+\left(\bar{z} D_{312}-4 c D_{212}\right) A_{2}+\left(\bar{z} D_{321}-4 c D_{221}\right) A_{3}+\left(\bar{z} D_{322}-4 c D_{222}\right) A_{4}=\frac{Q}{E I_{0}}
\end{aligned}
$$

at $(z=L)$

$$
\begin{aligned}
&\left(\bar{z}^{a_{1}} D_{21}-2 a_{1} c \bar{Z}^{\left(a_{1}-1\right)} D_{11}+a_{1}\left(a_{1}-1\right) c^{2} \bar{z}^{\left(a_{1}-2\right)} D_{01}\right) A_{1} \\
&+\left(\bar{z}^{a_{1}} D_{22}-2 a_{1} c \bar{z}^{\left(a_{1}-1\right)} D_{12}+a_{1}\left(a_{1}-1\right) \bar{Z}^{\left(a_{1}-2\right)} c^{2} D_{02}\right) A_{2} \\
&+\left(\bar{z}^{a_{2}} D_{21}-2 a_{2} c \bar{z}^{\left(a_{2}-1\right)} D_{11}+a_{2}\left(a_{2}-1\right) c^{2} \bar{z}^{\left(a_{2}-2\right)} D_{01}\right) A_{3} \\
&+\left(\bar{z}^{a_{2}} D_{22}-2 a_{2} c \bar{Z}^{\left(a_{2}-1\right)} D_{12}+a_{2}\left(a_{2}-1\right) c^{2} \bar{z}^{\left(a_{2}-2\right)} D_{02}\right) A_{4}=0 \\
&\left(\bar{z} D_{311}-4 c D_{211}\right) A_{1}+\left(\bar{z} D_{312}-4 c D_{212}\right) A_{2}+\left(\bar{z} D_{321}-4 c D_{221}\right) A_{3}+\left(\bar{z} D_{322}-4 c D_{222}\right) A_{4}=0
\end{aligned}
$$

where:

$$
\left.\begin{array}{l}
D_{01}=\sin \left(\frac{\bar{\beta}}{a} \ln (\bar{z})\right) \\
D_{02}=\cos \left(\frac{\bar{\beta}}{a} \ln (\bar{z})\right) \\
D_{11}=-\frac{\bar{\beta}}{a}\left(\frac{c}{\bar{z}}\right) \cos \left(\frac{\bar{\beta}}{a} \ln (\bar{z})\right) \\
D_{12}=\frac{\bar{\beta}}{a}\left(\frac{c}{\bar{z}}\right) \sin \left(\frac{\bar{\beta}}{a} \ln (\bar{z})\right) \\
D_{21}=-\gamma^{2} \sin \left(\frac{\bar{\beta}}{a} \ln (\bar{z})\right)-\frac{\bar{\beta}}{a}\left(\frac{c}{\bar{z}}\right)^{2} \cos \left(\frac{\bar{\beta}}{a} \ln (\bar{z})\right) \\
D_{22}=-\gamma^{2} \cos \left(\frac{\bar{\beta}}{a} \ln (\bar{z})\right)+\frac{\bar{\beta}}{a}\left(\frac{c}{\bar{z}}\right)^{2} \sin \left(\frac{\bar{\beta}}{a} \ln (\bar{z})\right)
\end{array}\right\}
$$

and

$$
\left.\begin{array}{l}
D_{211}=\bar{z}^{a_{1}} D_{21}-2 a_{1} c \bar{z}^{\left(a_{1}-1\right)} D_{11}+a_{1}\left(a_{1}-1\right) c^{2} \bar{z}^{\left(a_{1}-2\right)} D_{01} \\
D_{212}=\bar{z}^{a_{1}} D_{22}-2 a_{1} c \bar{z}^{\left(a_{1}-1\right)} D_{12}+a_{1}\left(a_{1}-1\right) c^{2} \bar{z}^{\left(a_{1}-2\right)} D_{02} \\
D_{221}=\bar{z}^{a_{2}} D_{21}-2 a_{2} c \bar{z}^{\left(a_{2}-1\right)} D_{11}+a_{2}\left(a_{2}-1\right) c^{2} \bar{z}^{\left(a_{2}-2\right)} D_{01} \\
D_{222}=\bar{z}^{a_{2}} D_{22}-2 a_{2} c \bar{z}^{\left(a_{2}-1\right)} D_{12}+a_{2}\left(a_{2}-1\right) c^{2} \bar{z}^{\left(a_{2}-2\right)} D_{02} \\
D_{311}=\bar{z}^{a_{1}} D_{31}-3 a_{1} c \bar{z}^{\left(a_{1}-1\right)} D_{21}+3 a_{1}\left(a_{1}-1\right) c^{2} \bar{z}^{\left(a_{1}-2\right)} D_{11}-a_{1}\left(a_{1}-1\right)\left(a_{1}-2\right) c^{3} \bar{z}^{\left(a_{1}-3\right)} D_{01} \\
D_{312}=\bar{z}^{a_{1}} D_{32}-3 a_{1} c \bar{z}^{\left(a_{1}-1\right)} D_{22}+3 a_{1}\left(a_{1}-1\right) c^{2} \bar{z}^{\left(a_{1}-2\right)} D_{12}-a_{1}\left(a_{1}-1\right)\left(a_{1}-2\right) c^{3} \bar{z}^{\left(a_{1}-3\right)} D_{02} \\
D_{321}=\bar{z}^{a_{2}} D_{31}-3 a_{2} c \bar{z}^{\left(a_{2}-1\right)} D_{21}+3 a_{2}\left(a_{2}-1\right) c^{2} \bar{z}^{\left(a_{2}-2\right)} D_{11}-a_{2}\left(a_{2}-1\right)\left(a_{2}-2\right) c^{3} \bar{z}^{\left(a_{2}-3\right)} D_{01} \\
D_{322}=\bar{z}^{a_{2}} D_{32}-3 a_{2} c \bar{z}^{\left(a_{2}-1\right)} D_{22}+3 a_{2}\left(a_{2}-1\right) c^{2} \bar{z}^{\left(a_{2}-2\right)} D_{12}-a_{2}\left(a_{2}-1\right)\left(a_{2}-2\right) c^{3} \bar{z}^{\left(a_{2}-3\right)} D_{02}
\end{array}\right\}
$$


in which,

$$
\left.\begin{array}{c}
D_{31}=\gamma^{3} \cos \left(\frac{\bar{\beta}}{a} \ln (\bar{z})\right)-2\left(\frac{\bar{\beta}}{a}\right)^{2}\left(\frac{c}{\bar{z}}\right)^{3} \sin \left(\frac{\bar{\beta}}{a} \ln (\bar{z})\right)-\gamma^{2}\left(\frac{c}{\bar{z}}\right) \sin \left(\frac{\bar{\beta}}{a} \ln (\bar{z})\right)+2 \frac{\bar{\beta}}{a}\left(\frac{c}{\bar{z}}\right)^{3} \cos \left(\frac{\bar{\beta}}{a} \ln (\bar{z})\right) \\
D_{32}=-\gamma^{3} \sin \left(\frac{\bar{\beta}}{a} \ln (\bar{z})\right)-2\left(\frac{\bar{\beta}}{a}\right)^{2}\left(\frac{c}{\bar{z}}\right)^{3} \cos \left(\frac{\bar{\beta}}{a} \ln (\bar{z})\right)-\gamma^{2}\left(\frac{c}{\bar{z}}\right) \cos \left(\frac{\bar{\beta}}{a} \ln (\bar{z})\right)+2 \frac{\bar{\beta}}{a}\left(\frac{c}{\bar{z}}\right)^{3} \sin \left(\frac{\bar{\beta}}{a} \ln (\bar{z})\right) \\
\gamma=\left(\frac{\bar{\beta}}{a}\right)\left(\frac{c}{\bar{z}}\right)
\end{array}\right\}
$$

\section{Numerical Examples}

In this paper, the behavior of two groups of piles (with square cross-section) is investigated. The geometry, loading condition and materials constants for each group are presented in Figure 2 and Figure 3.

The lateral deflection, the distribution of shear force and bending moment along the pile shaft are given in Figures 4-6.

The results of group No. 2 are given in Figures 7-9. Pile (S2) in this group has the same material volume for pile (T3).

It can be observed from Figure 4 and Figure 7 that tapered piles show stiffer behavior than prismatic ones having the same material volume. The deflection decrease for a tapering angle $\left(0.955^{\circ}\right)$ (pile T1) is found to be

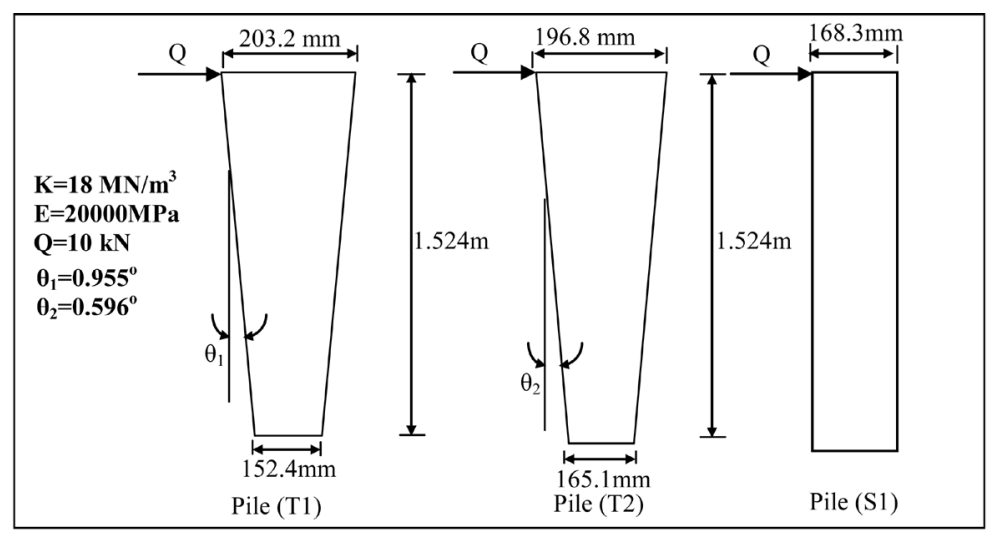

Figure 2. Pile group No. 1.

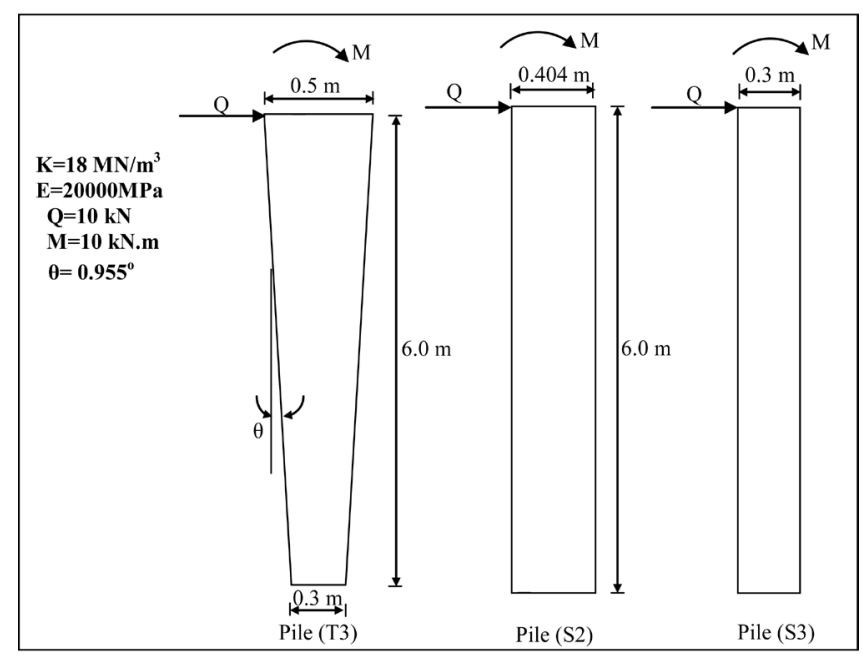

Figure 3. Pile group No. 2. 
M. A. Qissab

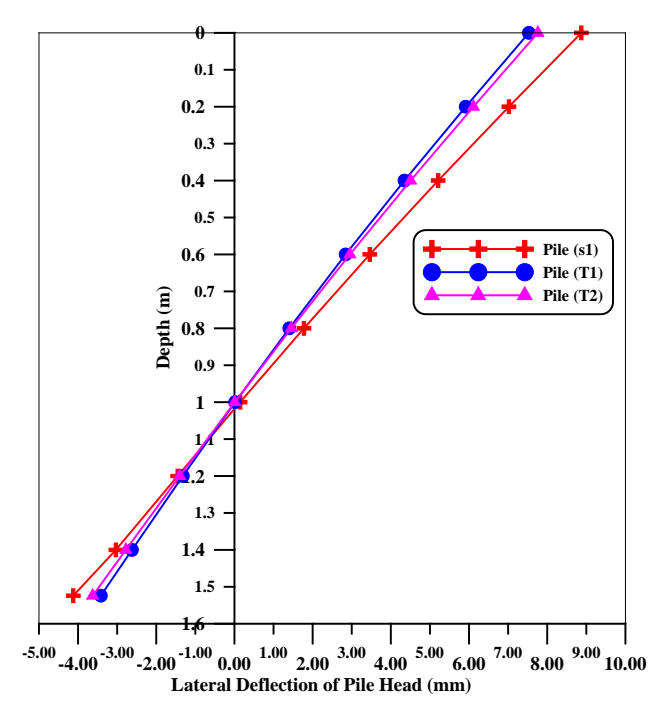

Figure 4. Lateral deflection of pile group No. 1.

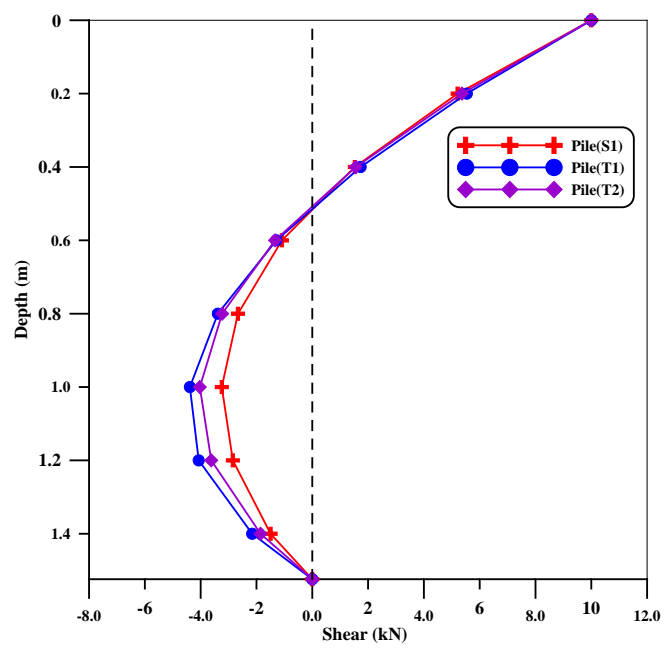

Figure 5. Shear force distribution for pile group No. 1.

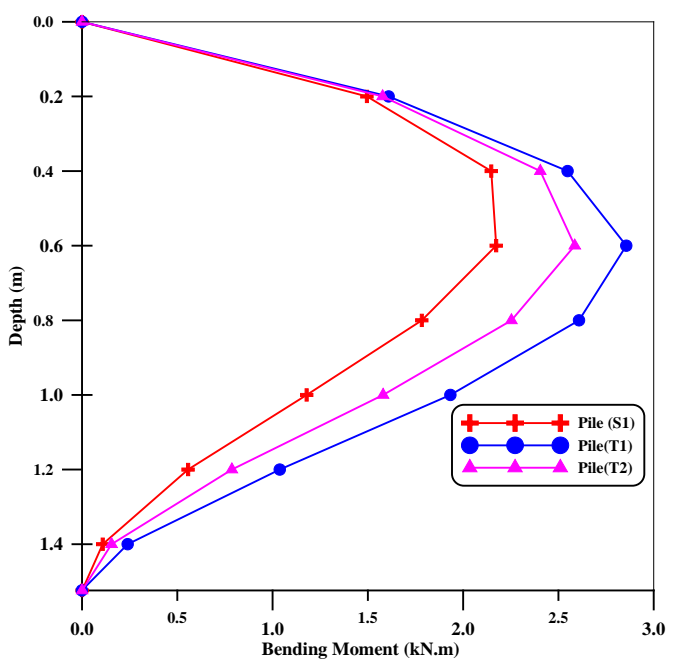

Figure 6. Moment distribution for pile group No. 1.

34 


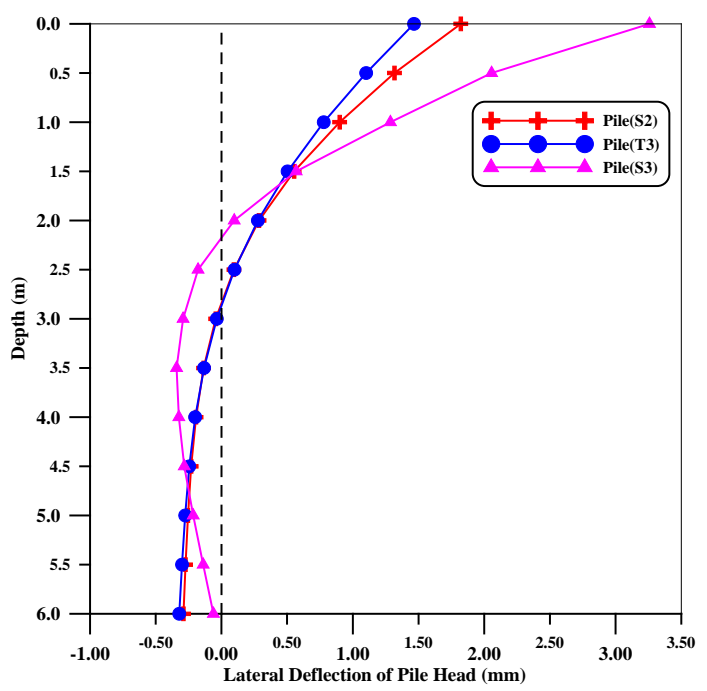

Figure 7. Lateral deflection for pile group No. 2.

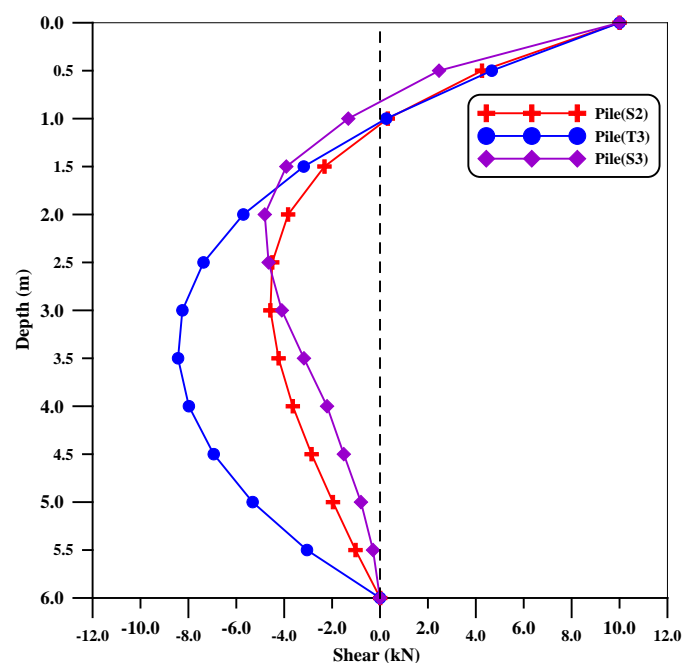

Figure 8. Shear force distribution for pile group No. 3.

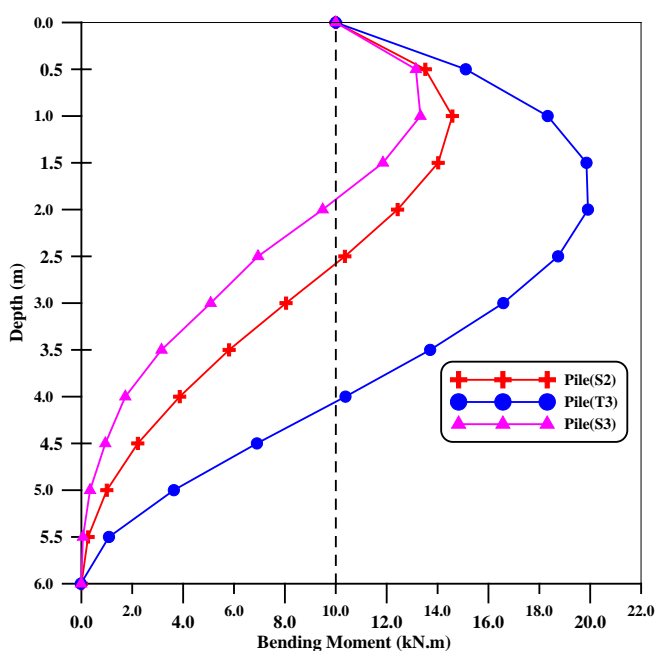

Figure 9. Moment distribution for pile group No. 3. 
(17.84\%) for group No. 1 and (22\%) for group No. 2 (pile T3) for the same material volume. Also, it can be noted that the point of maximum bending moment is located approximately at the upper third of the pile depth at which the cross-sectional area of the tapered piles is larger than that for prismatic ones having the same material volume. This gives a more efficient material distribution.

\section{Verification of the Present Solution}

To verify the obtained results from the derived equations and because there is no laboratory studies that concerned with laterally loaded tapered piles in cohesive soils, the finite element method is used to analyze piles T1 and T3 of the two groups. Each pile is subdivided into a number (10) of straight elements as shown in Figure 10 and a computer program is used to solve the problem.

It can be noted from the Figure 11 and Figure 13 that the lateral deflection curves obtained from the two solutions are identical. On the other hand, the moment distribution curves shown in Figure 12 and Figure 14 obtained by the finite element method give a lower-bound solution for the bending moment. This is due to the lack in shear diagram resulting from subdividing the pile into a number of straight elements which cause a loss in bending moment (the area of the shear diagram). To obtain better results for the bending moment, finer divisions for the pile should be used. This verifies the accuracy and efficiency of the proposed solution in comparison to the finite element method.

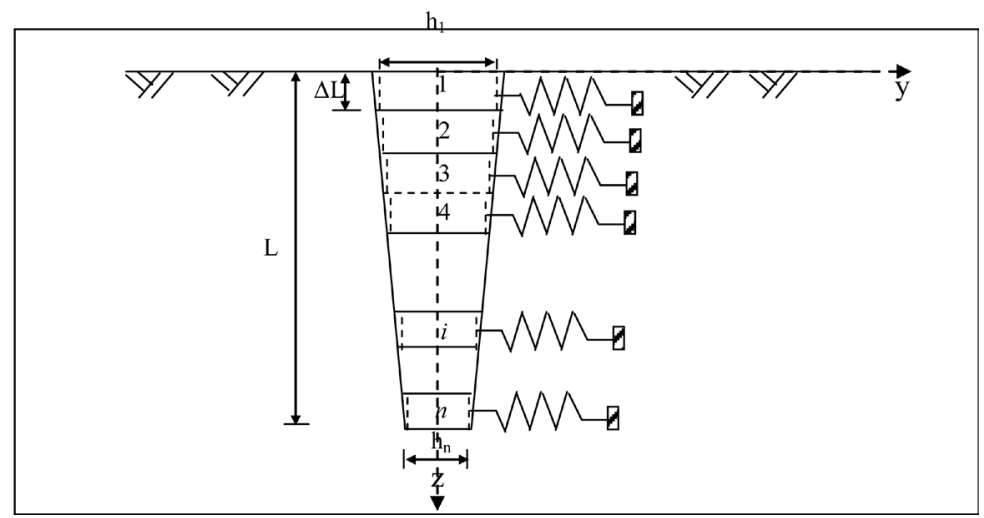

Figure 10. The proposed finite element model of the tapered pile.

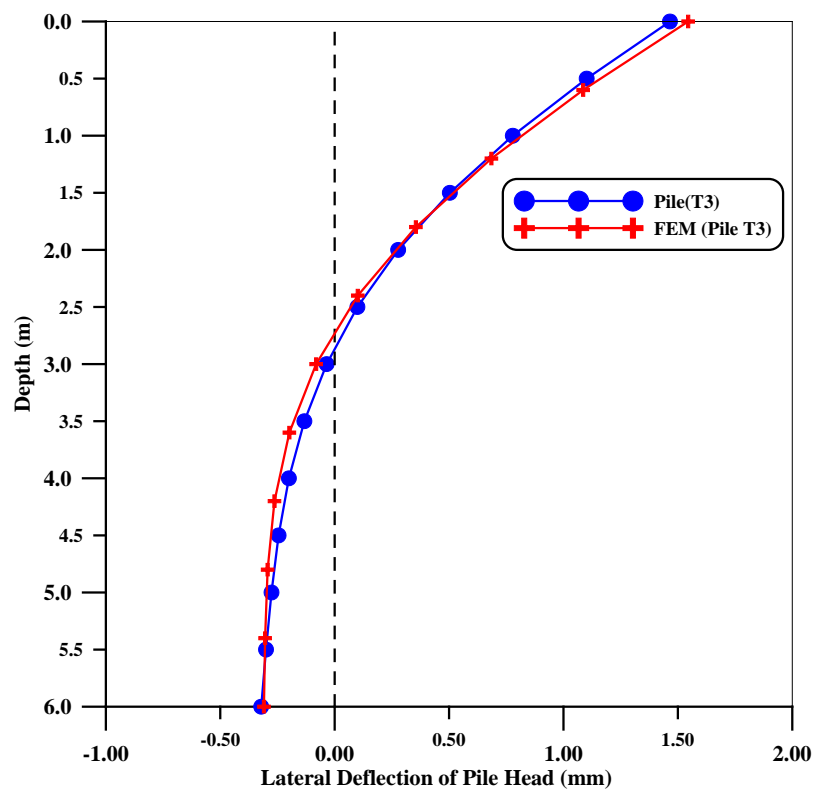

Figure 11. Lateral deflection for piles T4 and S4. 
M. A. Qissab

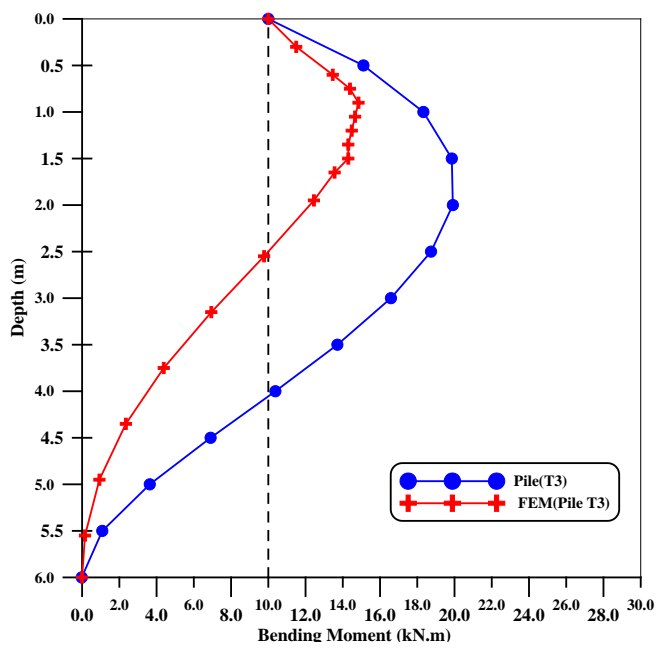

Figure 12. Moment distribution for piles T4 and S4.

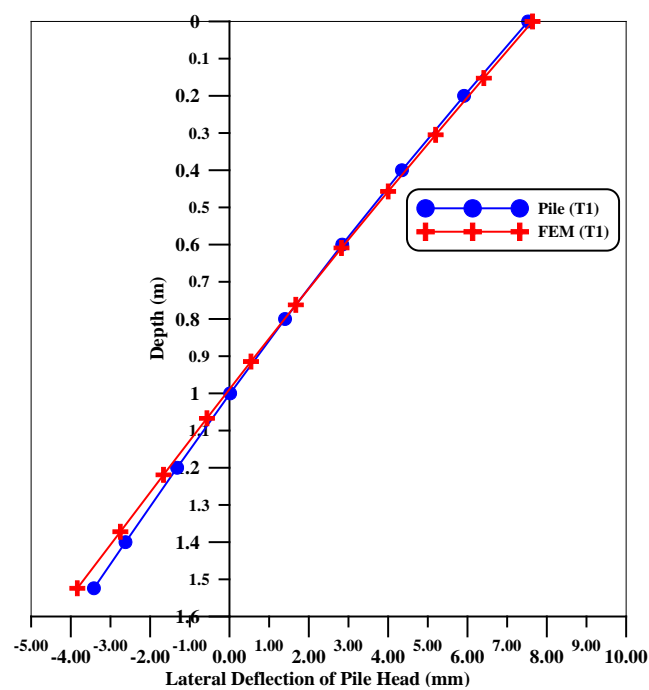

Figure 13. Lateral deflection for pile T1.

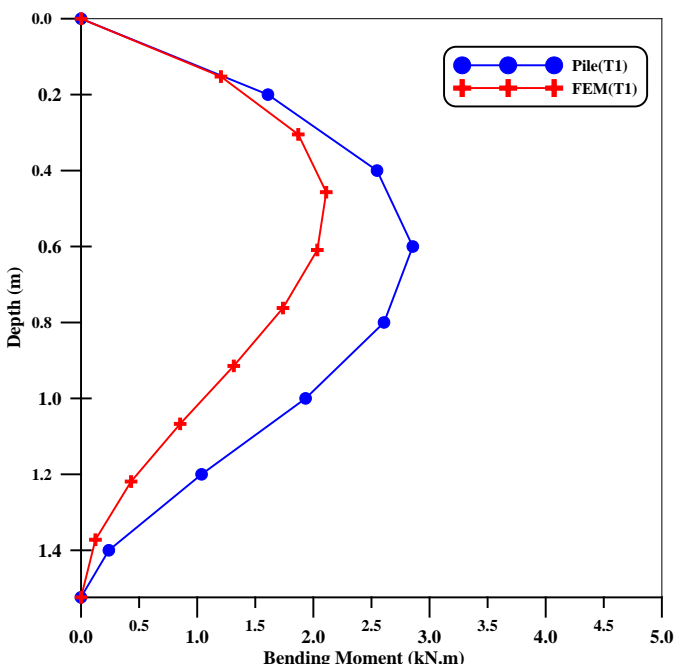

Figure 14. Moment distribution for pile T1.

37 


\section{Conclusion}

In this paper, an exact solution of the differential equation for a pyramidal tapered pile with square cross-sectional area in cohesive soils has been obtained based on beam-on-elastic foundation theory assuming constant value for the subgrade reaction coefficient. It is clear from the presented results that tapered piles show stiffer behavior than that for prismatic ones having the same material volume. It is found that the decrease in deflection for a tapering angle $0.955^{\circ}$ is in the range of $17.84 \%-22 \%$ for the studied cases. The value of the maximum bending moment for tapered piles is found to be larger than that for prismatic piles. The increase in maximum bending moment for tapered piles is proportional to the increase in cross-section size which gives approximately constant bending stress. As a result, tapered piles are more efficient and economic than those having the same material volume.

\section{References}

[1] Wei, J.Q. (1998) Experimental Investigation of Tapered Piles. M.Sc. Thesis, University of Western Ontario, London, Canada.

[2] Horvath, J.S., Trochalides, T., Burns, A. and Merjan, S. (2004) A New Type of Tapered Steel Pipe Pile for Transportation Applications. Geo-Trans, American Society of Civil Engineers Geo-Institute, California.

[3] Shanker, K., Basudhar, P.K. and Patra, N.R. (2008) Flexural Response of Tapered Piles in Liquefied Soils. The 12th International Conference of International Association for Computer Methods and Advances in Geomechanics (IACMAG), Goa.

[4] Zhan, Y., Wang, H. and Liu, F. (2012) Numerical Investigation on Load Capacity Behavior of Tapered Pile Foundations. EJGE, 17, 1969-1980.

[5] Hetenyi, M. (1946) Beams on Elastic Foundations. University of Michigan Press, Ann Arbor. 
Scientific Research Publishing (SCIRP) is one of the largest Open Access journal publishers. It is currently publishing more than 200 open access, online, peer-reviewed journals covering a wide range of academic disciplines. SCIRP serves the worldwide academic communities and contributes to the progress and application of science with its publication.

Other selected journals from SCIRP are listed as below. Submit your manuscript to us via either submit@scirp.org or Online Submission Portal.
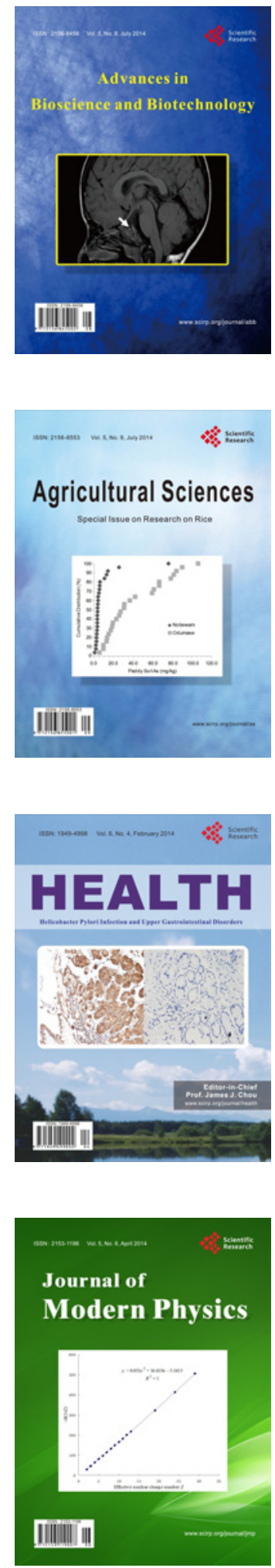
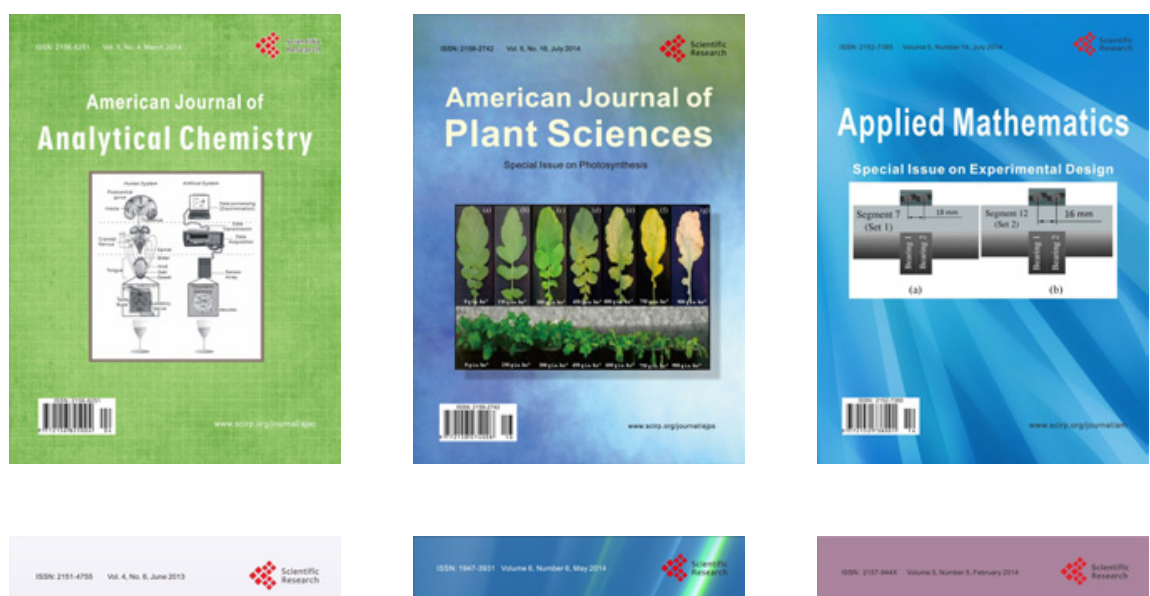

Creative Education
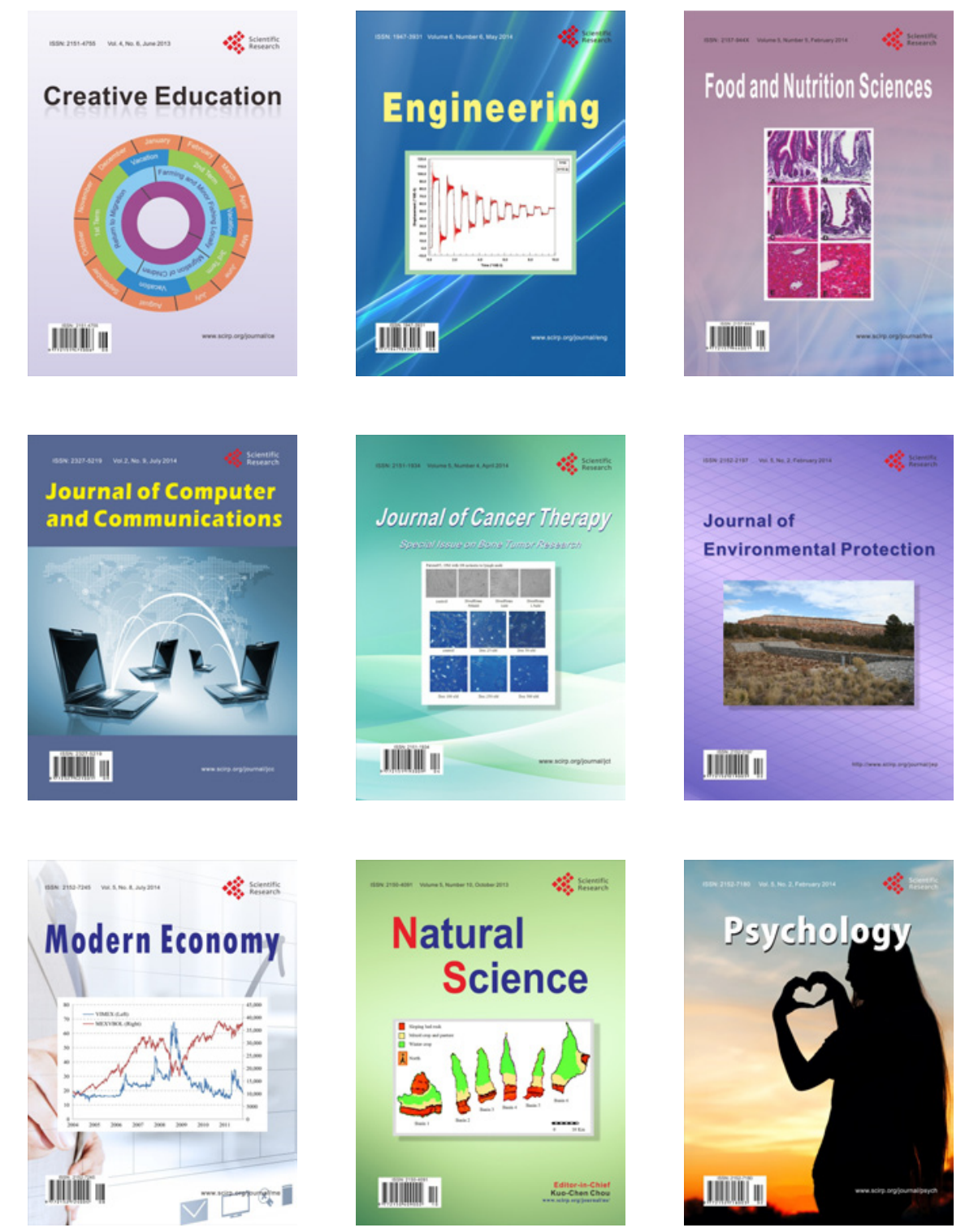